\title{
CURRENT STATUS OF DIII-D REAL-TIME DIGITAL PLASMA CONTROL
}

\author{
by \\ B.G. PENAFLOR, D.A. PIGLOWSKI, J.R. FERRON, \\ and M.L. WALKER
}

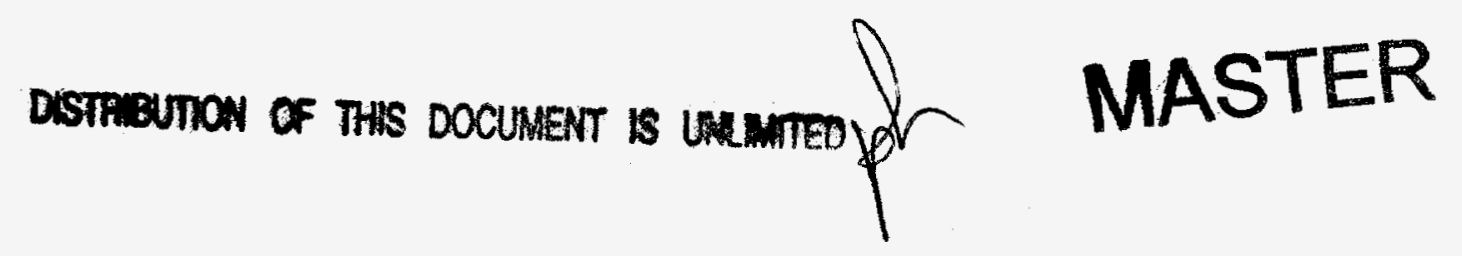

JUNE 1999 


\section{DISCLAIMER}

This report was prepared as an account of work sponsored by an agency of the United States Government. Neither the United States Government nor any agency thereof, nor any of their employees, makes any warranty, express or implied, or assumes any legal liability or responsibility for the accuracy, completeness, or usefulness of any information, apparatus, product, or process disclosed, or represents that its use would not infringe privately owned rights. Reference herein to any specific commercial product, process, or service by trade name, trademark, manufacturer, or otherwise does not necessarily constitute or imply its endorsement, recommendation, or favoring by the United States Government or any agency thereof. The views and opinions of authors expressed herein do not necessarily state or reflect those of the United States Government or any agency thereof. 


\section{DISCLAIMER}

Portions of this document may be illegible in electronic image products. Images are produced from the best available original document. 


\title{
CURRENT STATUS OF DIII-D REAL-TIME DIGITAL PLASMA CONTROL
}

\author{
by \\ B.G. PENAFLOR, D.A. PIGLOWSKI, J.R. FERRON, \\ and M.L. WALKER
}

This is a preprint of a paper to be presented at the 11th IEEE/NPSS Real Time Conference, June 14-18, 1999, Santa Fe, New Mexico and to be published in Transactions on Nuclear Science.

\author{
Work supported by \\ the U.S. Department of Energy \\ under Contract No. DE-AC03-99ER54463
}




\title{
Current Status of DIII-D Real-Time Digital Plasma Control ${ }^{*}$
}

\author{
B.G. Penaflor, D.A. Piglowski, J.R. Ferron, and M.L. Walker \\ General Atomics, P.O. Box 85608, San Diego, California 92186-5608
}

\begin{abstract}
This paper describes the current status of real-time digital plasma control for the DIII-D tokamak. The digital plasma control system (PCS) has been in place at DIII-D since the early 1990 s and continues to expand and improve in it's capabilities to monitor and control plasma parameters for DIII-D fusion science experiments. The PCS monitors over 200 tokamak parameters from the DIII-D experiment using a real-time data acquisition system that acquires a new set of samples once every $60 \mu \mathrm{s}$. This information is then used in a number of feedback control algorithms to compute and control a variety of parameters including those affecting plasma shape and position.
\end{abstract}

A number of recent improvements to the control capabilities of the PCS along with general system enhancements have contributed to the advancement and understanding of fusion energy science at DIII-D. Among the more significant improvements to the PCS control capabilities has been the successful incorporation of advanced techniques for reconstruction of plasma equilibrium parameters in realtime. An isoflux control method using these improved plasma parameter calculations has greatly enhanced the ability of achieving desired shaped plasma on the DIII-D tokamak and even made it possible to achieve new and different ones.

A number of system related improvements has improved the usability and flexibility of the DIII-D PCS. These include more graphical user interfaces to assist in entering and viewing the large and ever growing number of parameters controlled by the PCS, increased interaction and accessibility from other DIII-D applications, and upgrades to the computer hardware and vended software.

Future plans for the system include possible upgrades of the real-time computers, further links to other DIII-D diagnostic measurements such as real-time Thomson scattering analysis, and joint collaborations with other tokamak experiments including the NSTX at Princeton.

\section{INTRODUCTION}

A major emphasis of the General Atomics' DIII-D fusion research program is to improve knowledge and understanding in the science of plasmas in which fusion reactions can occur. The DIII-D tokamak [1] is used to generate plasmas through means of magnetic confinement for scientific study. Due to their fluid nature, plasmas produced in tokamaks cannot be maintained without active real-time feedback control. At DIII-D much effort has gone into designing and developing better methods for maintaining and controlling the plasmas produced during tokamak discharges. A real-time digital plasma control system has been in place at the DIII-D tokamak fusion experiment facility since the early 1990s. This system referred to commonly as the PCS plays a vital role in fusion research at DIII-D providing scientists and engineers with a powerful and flexible set of tools for specifying and controlling the parameters for plasmas produced.

The primary function of the PCS is to perform the realtime feedback control of the plasma shape and position. A real-time computer system employing six Intel i860 processors working in parallel digitizes 224 signals from tokamak diagnostics and sends back commands to magnetic field coil power supplies which directly affect the plasma behaviour. Control of the plasma is for the most part performed by comparing user input plasma target values with estimates of actual ones calculated from the tokamak diagnostics. The difference between the target and actual values produces an error term which is used to derive the necessary commands to send back to the tokamak hardware in order to bring the plasma parameters closer to the desired targets. Examples of targets which can be specified for control by the PCS include the plasma's vertical position within the vessel, the distance of the plasma from the vessel walls and the location in which the plasma power is directed to strike the walls.

A secondary function of the DIII-D PCS is the control of hardware influencing other aspects of the discharge not directly related to plasma shape and position. This includes gas puffing into the tokamak to control the density, modulation of neutral beam heating sources and outputs from rf antennas. In addition to these control functions, the PCS also computes important information used in analyzing the performance of the plasma after each discharge.

\section{SYSTEM ARCHITECTURE AND DESIGN}

Computing hardware (Fig. 1) for the PCS consists of six Intel i860 VME based processors for performing the real-time feedback control, a VME Force5 Sun host computer running Solaris 2.6 OS which processes and loads discharge information specified by the users into the $i 860$ computers and a recently purchased Sun Ultra Sparc 10 also running Solaris 2.6 OS which is responsible for running the user interfaces and the associated server process for managing all of the discharge input parameters. The data acquisition hardware consists of a set of DSP Technology TRAQ digitizers which interface to the real-time computers through GA designed and manufactured data acquisition boards which are capable of writing a new set of digitized information into the memory of

\footnotetext{
*Work supported by U.S. Department of Energy under Contract No. DE-AC03-99ER54463.
} 


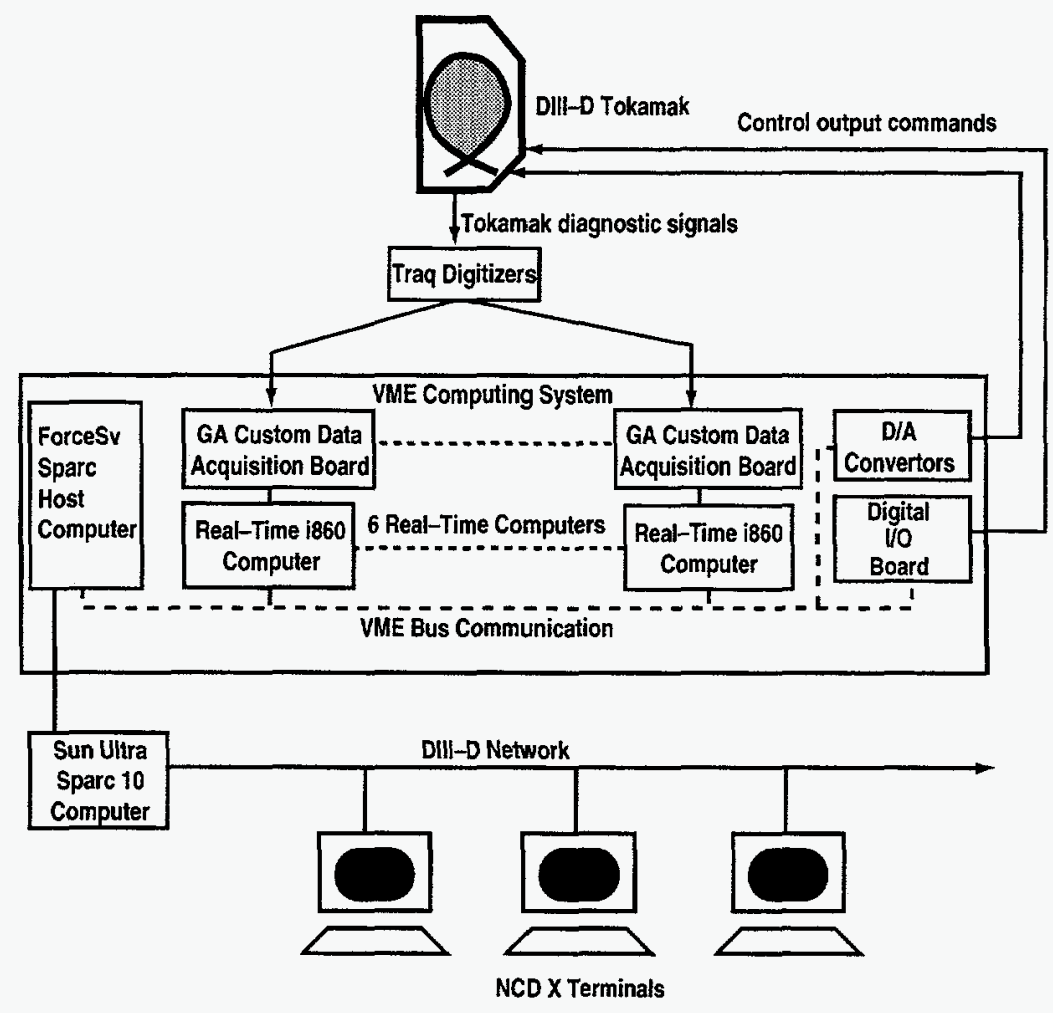

Fig. 1. PCS computing system hardware architecture.

the i860 computers at a rate of once every $10 \mu \mathrm{s}$. The hardware for sending the outputs from the PCS to the tokamak consists of five DATEL Inc., eight channel D/A converters and a single VME Microsystems Digital I/O board.

The PCS software application consists of a graphical user interface written in the IDL programming language and several programs written in $\mathrm{C}$ and $\mathrm{I} 860$ assembly. The $\mathrm{C}$ and assembly codes provide the important functions of managing the large number of plasma discharge parameters, synchronizing the PCS with the DIII-D discharge cycle, loading information from the user interfaces into the realtime computers, performing the realtime feedback control, and archiving the data generated after each discharge.

The PCS application has been designed to fit the specific manner in which experiments are performed on the DIII-D tokamak. The PCS user interface can be invoked and used by several users at a time from any $\mathrm{X} 11$ Windows client with privileged access to the DIII-D PCS computer systems. During DIII-D Plasma operations up to six or more physicists and engineers work together to program the large number of parameters to specify each discharge, each one using a separate copy of the PCS user interface. The inputs from these interfaces are managed by a PCS tcp/ip server process known as the "waveform server." This process queues and processes the requests from users to view and alter the discharge parameters.

A PCS "lockserver" process coordinates with the primary tokamak control computer to synchronize all of the PCS processes with the DIII-D discharge cycle. A typical discharge cycle runs about fifteen minutes during which time the setups for the discharge occur, a plasma pulse is run, data is acquired and analysis of the discharge results is performed.

Minutes prior to each plasma pulse the PCS enters a "lockout" state in which changes from users which would affect the upcoming discharge are "locked out" or kept from being used. Upon entering a lockout state a set of PCS processes running on the host computer obtains the latest discharge parameters from the PCS waveform server, processes the information and loads it into the memory of the realtime computers. When the tokamak hardware and computers have completed all of the necessary setups a plasma discharge is produced. The duration of a typical plasma discharge in which the PCS computers are also actively performing realtime feedback control on the plasma lasts for under ten seconds. After the discharge the PCS host processes write all of the associated information into the DIII-D archives and the PCS is unlocked and becomes ready to accept inputs for the next discharge.

\section{BENEFITS OF THE DIII-D PCS}

Major benefits of the system include a powerful and easy to use graphical user interface for viewing and modifying hundreds of parameters required for each plasma discharge, flexibility and ease of incorporating new control capabilities, full integration into the rest of the DIII-D computing environment, and the ability to easily view and restore parameters from past discharges in whole or in part into setups for future discharges. 


\section{A. User Interface}

Each plasma discharge requires a team of physicists programming literally hundreds of parameters affecting the outcome of the discharge as well as the outcome of the related physics experiments. The PCS provides a well organized framework and all of the necessary tools for easily specifying these parameters. The PCS graphical user interface (Fig. 2) provides physicists with a fast and simple way of selecting, viewing and editing time dependent waveform data.

The inputs into the PCS for each discharge consist primarily of time dependent waveform data. Most of these are used to specify target values for controlling quantities such as power supply voltages and currents, gas outputs, neutral beam duty cycles, plasma vertical position and distances from the vessel walls and more. Other waveform information is also available for specifying a wide range of values dependent upon the discharge time including inputs such as choice of control techniques, and gain values used in converting calculated error values into output commands. A large portion of the PCS user interface is dedicated to displaying and providing a means for easily inserting and editing waveform data. The PCS user interface contains menus for selecting waveforms to appear for display and edit in a two dimensional plot grid window showing desired values plotted against discharge time. Waveforms can be edited using "point and click" technology directly in the plot window or by entering numeric vertex values in a numerics mode input screen. The PCS also provides tools for easily entering special types of waveforms including sine, triangle and square waveforms.

A second type of input to the PCS are static pieces of information typically associated with specific control methods which for the most part remain constant throughout a discharge and are not dependent upon the discharge time.

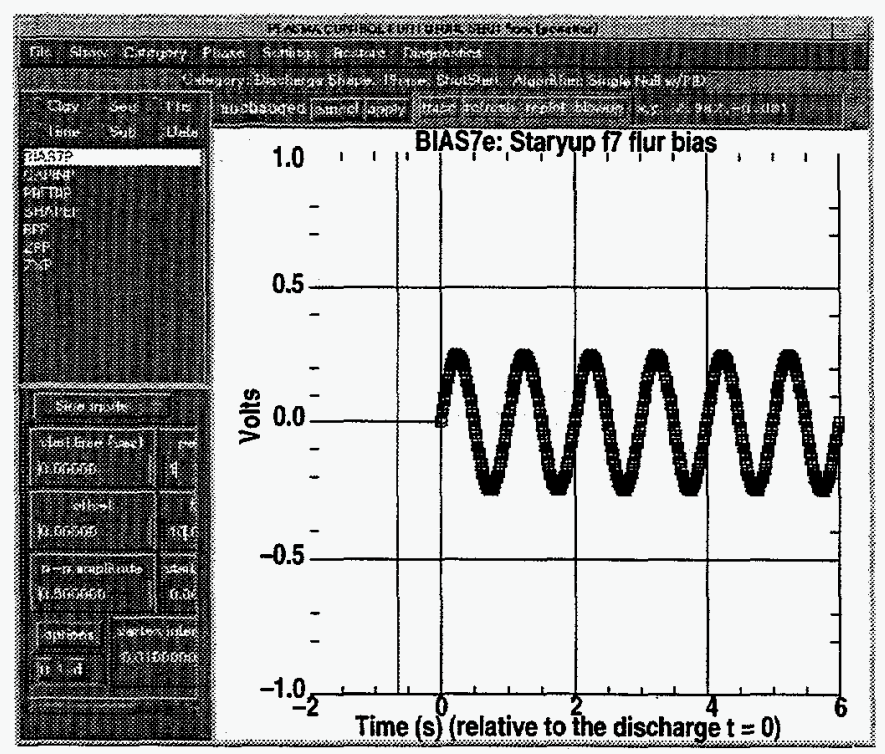

Fig. 2. PCS user interface.
These static data inputs include many of the parameters required by the isoflux control method [2] such as the basis shape, grid and segment definitions used by the PCS to set up for this special advanced type of control of the plasma shape and position. The definition, quantity and means of editing this class of static inputs is flexible and depends upon the needs of the control methods they are associated with. In the case of the static inputs needed by the isoflux control a special basis shape editor displaying a cross section of the DIII-D vessel and plasma was developed to aid users in visualizing the desired shape.

\section{B. System Flexibility}

Use of the programmable digital computer system for DIII-D plasma control has provided greater flexibility in optimizing use of the present equipment by providing software alternatives to problems which otherwise would have required the purchase or construction of new hardware. A recent example of this was the implementation of a software solution for protecting some in-vessel hardware in the DIII-D tokamak from overheating. Because of time constraints a hardware solution was not feasible. Instead, an algorithm was installed in the PCS which continuously monitors the discharge and actively keeps the discharge properly positioned.

\section{Integration into DIII-D Computing Environment}

The PCS has been fully integrated into the computing environment at DIII-D through means of tcp/ip client/server codes developed at GA which allow the PCS to coordinate and share information with the rest of the computer systems at DIII-D. These include the DIII-D PTDATA [3] archiving system, the tokamak control computer, the main DIII-D data acquisition computer, and the neutral beam computers. Examples of this integration include synchronization with the tokamak control computer during a discharge cycle, and sharing of parameters programmed by users of the PCS such as the plasma current and density.

\section{Restoring Parameters used in Past Discharges}

One of the most important and often used features provided by the DIII-D PCS is the ability to restore and reuse all or parts of the parameters used in past discharges. All information used by the PCS to specify a discharge is saved into an archive file with an associated number uniquely identifying the discharge. The archive files are permanently stored into the DIII-D PTDATA archive system and can be recalled at any time in the future. A special PCS user interface provides a means for users to easily recall past discharge setup information and select any part of that information to load into setups for new discharges. The PCS interface allows restores ranging from vertices for individual waveforms all the way to the entire setup information for a past discharge. 


\section{RECENT IMPROVEMENTS TO THE PCS}

Several improvements to the PCS have been made within the past year. Among these has been a number of enhancements to the basic control capabilities, software improvements to the PCS application and upgrades of hardware and software.

Work to improve PCS control over the power supply current regulators to enable control algorithms used in the PCS to send out known output voltages to these power supplies was recently concluded and made operational. Before completion of this work the PCS was limited only to the ability to either increase or decrease them [4].

The first phase of implementation has begun on a method for performing simultaneous manipulation of all coil commands sent by the PCS as a function of all shape error signals [5]. Past methods for computing individual control commands to coils relied on one or at most two closely related signals. The new multiple input multiple output control method provides more coordinated control which would prevent commands for one set of coils from interfering with commands for another.

Much extensive work has been done to improve the new methods for controlling the plasma known as "isoflux" control. The isoflux method controls magnetic flux targets at designated poloidal "control points" as oppossed to past methods designed to control estimates of plasma shape and position targets. Improvements in the accuracy of plasma shape estimation defined by flux contours have been made through the use of equilibrium reconstruction techniques [6] now performed in real-time by the PCS. By taking advantage of the more accurate flux contour shape estimations the isoflux control method significantly improves and expands the range of control possibilities of the DIII-D PCS.

Improvements to neutral beam power injection were recently introduced with the development of PCS codes to monitor neutral beam heating sources in real-time and substitute failed beam sources with standby ones [7].

Improvements to the PCS software introduced recently by the PCS development staff include a more powerful graphical user interface for editing matrix data. The new matrix editor presents a spreadsheet like interface which allows users to easily view and edit table information and also make edits to multiple matrices at a time. Another software improvement to the PCS was made in the release of a message server for managing PCS status and error messages and a set of graphical user interface client windows for displaying the PCS messages to users in an organized format [8].

A new Sun Ultra Sparc 10 workstation has greatly improved performance of the user interfaces and also enhanced PCS software development by reducing the time to compile the entire PCS application from one hour to ten minutes. The operating systems for the Sun computers was upgraded to Solaris 2.6 in order to resolve y $2 \mathrm{k}$ compliance issues in the PCS.

\section{FUTURE PLANS}

The PCS has been very successful in providing the tools necessary for performing plasma shape control on the DIII-D tokamak and has demonstrated great flexibility in keeping up with the needs of the fusion research program. In anticipation of even higher demands on the capabilities of the PCS by the research program, planning for a PCS upgrade in computing hardware has begun. Among the possible new platforms being considered for the next generation PCS computer are the Compaq Alpha workstations which could potentially greatly increase performance of the realtime system improving shape estimation and control. In addition to this upgrade planning, the PCS development staff continues to support the current system to meet the present experimental requirements and improve the tools for users. Also a collaborative effort was recently begun with the Princeton Plasma Physics Laboratory to install a version of the DIII-D PCS for use in the NSTX experiment [9].

\section{REFERENCES}

[1] J.L. Luxon, L.G. Davis, Fusion Technol. 8, 441 (1985).

[2] J.R. Ferron, M.L. Walker, L.L. Lao, H.E. St.John, D.A. Humphreys, J.A. Leuer, "Real Time Equilibrium Reconstruction for Tokamak Discharge Control," Nucl. Fusion, Vol. 38, 1055 (1998).

[3] B.B McHarg Jr., "Access to DIII-D Data Located in Multiple Files and Multiple Locations," Proc. of the 15th IEEE/NPSS Symp. on Fusion Engineering, Vol. 1, 123 (1993).

[4] M.L. Walker, et al., "Status of DIII-D Plasma Control," Proc. 16th IEEE/NPSS Symp. on Fusion Engineering, Vol. 2, 885 (1995).

[5] M.L. Walker, D.A. Humphreys, J.R. Ferron, "Multivariable Shape Control Development on the DIII-D Tokamak," Proc. 17th IEEE/NPSS Symp. on Fusion Engineering, Vol. 1, 556 (1997)

[6] L.L. Lao, et al., Nucl. Fusion 251611 (1985).

[7] J.C. Phillips, "An Algorithm to Provide Real-Time Neutral Beam Substitution in The DIII-D Tokamak,"these proceedings.

[8] D.A. Piglowski, B.G. Penaflor, J.R. Ferron, "Interprocess Communication Within the DIII-D Plasma Control System," these proceedings.

[9] D. Gates, "NSTX Control System Development Plan," these proceedings. 